Bull. Austral. Math. Soc.

$16 \mathrm{~A} 65,16 \mathrm{~A} 22$

Vol. 39 (1989) [141-143]

\title{
THE ENDOMORPHISM RING OF A FINITE-LENGTH MODULE
}

\author{
RAINER Schulz
}

\begin{abstract}
Let $M$ be an $R$-module of finite length. For a simple $R$-module $A$, let $\ell_{A}$ denote the nuber of times the isomorphism type of $A$ appears in a composition chain of $M$, and let $\sigma$ denote the maximum of the $\ell_{A}, A$ ranging over all simple submodules of $M$. Let $S$ be the endomorphism ring of $M$. We show that the Loewy length of $S$ is bounded by $\sigma$.
\end{abstract}

It is well-known that the endomorphism ring $S$ of a finite-length module $M_{R}$ over any ring $R$ is semi-primary, that means the factor ring of $S$ modulo its radical $J$ is semisimple artinian, and $J$ is nilpotent. The smallest number $m$ with the property $J^{m}=0$ is called the Loewy length of $S$. Let $\ell$ denote the length of $M_{R}$. Then the estimate $m \leqslant \ell$ holds. According to a remark of Bourbaki ([1, Chapter 8 , Section 2 , exercise 3$]$ ), this result is due to $A$. Rosenberg.

For any simple module $A_{R}$, let $\ell_{A}$ be the number of times the isomorphism type of $A_{R}$ appears as a composition factor in a composition chain of $M_{R}$. Let $h$ denote the maximum of the numbers $\ell_{A}, A_{R}$ ranging over all simple $R$-modules. Improving the estimate given above, Smalø [4] showed that the inequality $m \leqslant h$ holds.

In this paper, we will prove the estimate $m \leqslant \sigma$, where $\sigma$ is the maximum of the numbers $\ell_{A}, A_{R}$ ranging only over all simple submodules of $M_{R}$. Note that all of the numbers $\ell_{A}, \ell, h, \sigma$ are invariants of $M_{R}$ by the Jordan-Hölder Theorem. An analogous result for infinite cardinals was proved in [3, Satz 4], under more general assumptions on $M_{R}$, including not only finite-length modules, but also certain semiartinian modules which have perfect endomorphism rings. As the methods in [3] are rather technical, it might be useful to provide a simple proof for the estimate $m \leqslant \sigma$ in the finite-length case. This is the aim of the present note.

ThEOREM. Let $M_{R}$ be a finite-length module over any ring $R$. Let $S$ be the endomorphism ring of $M_{R}$. Then the Loewy length of $S$ is bounded by the number $\sigma$.

Proof: We need the following lemma (compare [2, Lemma 4], which may be of interest in its own right.

Received 15 April 1988

Copyright Clearance Centre, Inc. Serial-fee code: 0004-9729/89 \$A2.00+0.00. 
LemMA. Let ${ }_{S} X_{R}$ be a bimodule, where $S$ is a semi-primary ring and $X_{R}$ has finite length. Let $X_{R}$ have a composition factor isomorphic to some simple module $A_{R}$. Then the socle of ${ }_{S} X$, considered as a right $R$-module, has also a composition factor isomorphic to $A_{R}$.

Proof: Let $J=\operatorname{Rad}(S)$. Recall that $\operatorname{Soc}\left({ }_{s} X\right)=\operatorname{ann}_{X}(J)$, where $\operatorname{ann}_{X}$ denotes the right annihilator in $X$. Choose $x \in X$ and $U \subseteq X$ with $A_{R}$ isomorphic to $x R / U$. Assume at first $\operatorname{ann}_{X}(J) \cap x R \nsubseteq U$. Then there are $R$-isomorphisms $A \cong\left(\operatorname{ann}_{X}(J) \cap x R+U\right) / U \cong \operatorname{ann}_{X}(J) \cap x R / \operatorname{ann}_{X}(J) \cap x R \cap U$, and the assertion follows. Assume now $\operatorname{ann}_{X}(J) \cap x R \subseteq U$. As $X_{R}$ is artinian, there is a finite subset $\left\{f_{1}, \ldots, f_{k}\right\}$ of $J$ such that $\operatorname{ann}_{X}(J) \cap x R=\operatorname{ann}_{X}\left(f_{1}, \ldots, f_{k}\right) \cap x R$. Then the map $g: x R \rightarrow \Pi_{i=1}^{k} f_{i} x R, g(x r)=\left(f_{1} x r, \ldots, f_{k} x r\right)$ has kernel $\operatorname{ann}_{X}(J) \cap x R \subseteq U$. Therefore, the image of $g$ has a composition factor isomorphic to $A_{R}$, hence one of the $f_{i} x$ and $J X$ have a composition factor isomorphic to $A_{R}$.

The Loewy length of $J X$, considered as a left $S$-module, is one less than that of ${ }_{s} X$. The Lemma follows by induction over the $S$-Loewy length of the bimodule in question.

Proof of The Theorem: Let $f$ be a nonzero element of $J^{m-1}, m$ denoting the Loewy length of $S$. Let $A_{R}$ be a simple submodule of $M / \operatorname{Ker}(f)$. As $M / \operatorname{Ker}(f)$ embeds in $M_{R}$, the module $A_{R}$, up to isomorphism, is a simple submodule of $M_{R}$.

For any bimodule $s X_{R}$ and any subset $T$ of $S$, again let $\operatorname{ann}_{X}(T)$ denote the right annihilator of $T$ in $X$. Note that ann $X(T)$ is a $S-R$-bimodule. By the choice of $f$, the inclusion $\operatorname{ann}_{M}\left(J^{m-1}\right) \subseteq \operatorname{Ker}(f)$ holds, thus $A_{R}$ is a composition factor of $M / \operatorname{ann}_{M}\left(J^{m-1}\right)$. Consider now the ascending Loewy chain (=chain of iterated socles) of ${ }_{S} M$, this is the chain $0=\operatorname{ann}_{M}\left(J^{0}\right) \subset \operatorname{ann}_{M}(J) \subset \ldots \subset \operatorname{ann}_{M}\left(J^{m-1}\right) \subset$ $\operatorname{ann}_{M}\left(J^{m}\right)=M$.

As $A_{R}$ appears in the top factor module of this chain, we conclude that $A_{R}$ is a composition factor of each $S-R$-bimodule $X_{i}=M / \operatorname{ann}_{M}\left(J^{i}\right), 0 \leqslant i \leqslant m-1$. By our Lemma, the module $\operatorname{ann}_{X_{i}}(J)$ has a composition factor isomorphic to $A_{R}$. Using the identity $\operatorname{ann}_{X_{i}}(J)=\operatorname{ann}_{M}\left(J^{i+1}\right) / \operatorname{ann}_{M}\left(J^{i}\right)$ and looking again at the ascending Loewy chain of $s M$, we see that $A_{R}$ appears at least $m$ times as a composition factor in $M_{R}$, and we conclude that $m \leqslant \ell_{A} \leqslant \sigma$.

\section{REFERENCES}

[1] N. Bourbaki, Algèbre: Modules et Anneaux semi-simples, Chap. 8 (Hermann, Paris, 1958).

[2] R. Schulz, 'The endomorphism ring of an artinian module whose homogeneous length is finite', Proc. Amer. Math. Soc. 86 (1982), 209-210.

[3] R. Schulq, 'Die absteigende Loewylänge von Endomorphismenringen', Manuscripta Math. 45 (1984), 107-113. 
[4] S.O. Smalø, 'A limit on the Loewy length of the endomorphism ring of a module of finite length', Proc. Amer. Math. Soc. 81 (1981), 164-166.

Department of Algebra, Combinatorics

and Analysis

Auburn University

Alabama 36849

United States of America 